PROCEEDINGS OF THE

AMERICAN MATHEMATICAL SOCIETY

Volume 126, Number 4, April 1998, Pages 951-956

S $0002-9939(98) 04625-5$

\title{
HIGH ORDER MOMENTS OF CHARACTER SUMS
}

\author{
TODD COCHRANE AND ZHIYONG ZHENG
}

(Communicated by Dennis A. Hejhal)

Abstract. We establish the upper bound

$$
\frac{1}{p-1} \sum_{\chi \neq \chi_{o}}\left|\sum_{x=a+1}^{a+B} \chi(x)\right|^{2 k} \ll_{\epsilon, k} p^{k-1+\epsilon}+B^{k} p^{\epsilon},
$$

with $p$ a prime and $k$ any positive integer, the sum being over all nonprincipal multiplicative characters $(\bmod p)$.

1.

In this paper we obtain upper bounds on the character sum

$$
\frac{1}{p-1} \sum_{\chi \neq \chi_{o}}\left|\sum_{x=a+1}^{a+B} \chi(x)\right|^{2 k}
$$

where $a, B$ and $k$ are positive integers, $p$ is a prime, $\chi$ runs through the set of multiplicative characters $(\bmod p)$, and $\chi_{o}$ is the principal character. We shall assume that $B<p$ and that the interval $a+1 \leq x \leq a+B$ does not contain a multiple of $p$. A trivial bound for the sum in (1) that follows directly from the Polya-Vinogradov inequality is

$$
\frac{1}{p-1} \sum_{\chi \neq \chi_{o}}\left|\sum_{x=a+1}^{a+B} \chi(x)\right|^{2 k} \ll p^{k}(\log p)^{2 k} .
$$

Let $\mathcal{B}$ be the cube

$$
\mathcal{B}=\left\{\mathbf{x} \in \mathbb{Z}^{2 k}: a+1 \leq x_{i} \leq a+B, 1 \leq i \leq 2 k\right\}
$$

of cardinality $|\mathcal{B}|=B^{2 k}$, and let $V$ be the set of integer solutions of the congruence

$$
x_{1} x_{2} \ldots x_{k} \equiv x_{k+1} x_{k+2} \ldots x_{2 k} \quad(\bmod p) .
$$

Then

$$
|\mathcal{B} \cap V|=\frac{1}{p-1} \sum_{x_{1}=a+1}^{a+B} \ldots \sum_{x_{2 k}=a+1}^{a+B} \sum_{\chi} \chi\left(x_{1} x_{2} \ldots x_{k} x_{k+1}^{-1} \ldots x_{2 k}^{-1}\right)
$$

Received by the editors February 25, 1996.

1991 Mathematics Subject Classification. Primary 11L40, $11 D 79$.

Key words and phrases. Character sums, congruences. 


$$
\begin{gathered}
=\frac{|\mathcal{B}|}{p-1}+\frac{1}{p-1} \sum_{\chi \neq \chi_{o}} \sum_{x_{1}=a+1}^{a+B} \ldots \sum_{x_{2 k}=a+1}^{a+B} \chi\left(x_{1} x_{2} \ldots x_{k} x_{k+1}^{-1} \ldots x_{2 k}^{-1}\right) \\
=\frac{|\mathcal{B}|}{p-1}+\frac{1}{p-1} \sum_{\chi \neq \chi_{o}}\left|\sum_{x=a+1}^{a+B} \chi(x)\right|^{2 k} .
\end{gathered}
$$

Thus, we have

$$
\frac{1}{p-1} \sum_{\chi \neq \chi_{o}}\left|\sum_{x=a+1}^{a+B} \chi(x)\right|^{2 k}=|\mathcal{B} \cap V|-\frac{|\mathcal{B}|}{p-1} .
$$

For $k=1$ it is plain that $|\mathcal{B} \cap V|=B$ and so (4) is just

$$
\frac{1}{p-1} \sum_{\chi \neq \chi_{o}}\left|\sum_{x=a+1}^{a+B} \chi(x)\right|^{2}=B-\frac{B^{2}}{p-1} .
$$

In particular, if $B<(p-1) / 2$, then

$$
\frac{B}{2} \leq \frac{1}{p-1} \sum_{\chi \neq \chi_{o}}\left|\sum_{x=a+1}^{a+B} \chi(x)\right|^{2}<B
$$

whence

$$
\min _{\chi \neq \chi_{o}}\left|\sum_{x=a+1}^{a+B} \chi(x)\right| \leq \sqrt{B}
$$

and

$$
\max _{\chi \neq \chi_{o}}\left|\sum_{x=a+1}^{a+B} \chi(x)\right| \geq \sqrt{B / 2} .
$$

For $k=2$ it was shown in the paper of Ayyad, Cochrane and Zheng ([1], Theorem 2) that

$$
\frac{1}{p-1} \sum_{\chi \neq \chi_{o}}\left|\sum_{x=a+1}^{a+B} \chi(x)\right|^{4} \ll B^{2} \log ^{2} p,
$$

and that, for $B<\sqrt{p}$,

$$
\frac{1}{p-1} \sum_{\chi \neq \chi_{o}}\left|\sum_{x=a+1}^{a+B} \chi(x)\right|^{4} \gg B^{2} \log B,
$$

whence

$$
\max _{\chi \neq \chi_{o}}\left|\sum_{x=a+1}^{a+B} \chi(x)\right| \gg \sqrt{B}(\log B)^{1 / 4} .
$$

For higher moments Montgomery and Vaughan ([4], Theorem 1) established

$$
\frac{1}{p-1} \sum_{\chi \neq \chi_{o}} \max _{B}\left|\sum_{x=a+1}^{a+B} \chi(x)\right|^{2 k} \ll p^{k},
$$

which is sharper, by a power of $\log p$, than what one obtains trivially from the Polya-Vinogradov inequality. The main result of this paper is the following 
Theorem. For positive integers $k$,

$$
\frac{1}{p-1} \sum_{\chi \neq \chi_{o}}\left|\sum_{x=a+1}^{a+B} \chi(x)\right|^{2 k} \ll_{\epsilon, k} p^{k-1+\epsilon}+B^{k} p^{\epsilon} .
$$

In particular, for intervals of length $B \gg p^{1-\frac{1}{k}}$ we have

$$
\frac{1}{p-1} \sum_{\chi \neq \chi_{o}}\left|\sum_{x=a+1}^{a+B} \chi(x)\right|^{2 k} \ll_{\epsilon, k} B^{k} p^{\epsilon} .
$$

It is significant to note that the validity of (14) for arbitrary $k$ and $B<p$ is equivalent to the upper bound

$$
\left|\sum_{x=a+1}^{a+B} \chi(x)\right| \ll_{\epsilon} B^{1 / 2} p^{\epsilon}
$$

for nonprincipal $\chi$, which on the assumption of the Grand Riemann Hypothesis is known to be true; see Montgomery and Vaughan ([3]). We note that for $k=1$ and $k=2$ the upper bounds in (5) and (9) are sharper than (13).

For intervals of short length we may improve on (13) by writing

$$
\begin{gathered}
\frac{1}{p-1} \sum_{\chi \neq \chi_{o}}\left|\sum_{x=a+1}^{a+B} \chi(x)\right|^{2 k} \leq \max _{\chi \neq \chi_{o}}\left|\sum_{x=a+1}^{a+B} \chi(x)\right|^{2 k-4} \frac{1}{p-1} \sum_{\chi \neq \chi_{o}}\left|\sum_{x=a+1}^{a+B} \chi(x)\right|^{4} \\
\ll B^{2} \log ^{2} p \max _{\chi \neq \chi_{o}}\left|\sum_{x=a+1}^{a+B} \chi(x)\right|^{2 k-4}
\end{gathered}
$$

and then inserting the upper bound of Burgess ([2]),

$$
\left|\sum_{x=a+1}^{a+B} \chi(x)\right| \ll_{\epsilon} B^{1-\frac{1}{r}} p^{\frac{r+1}{4 r^{2}}} \log p
$$

where $r$ is any positive integer $\geq 2$. For intervals of length $\ll p^{1 / 4}$ we just insert the trivial upper bound $\left|\sum_{x=a+1}^{a+B} \chi(x)\right| \leq B$. In summary, we have for $k \geq 3$,

$$
\frac{1}{p-1} \sum_{\chi \neq \chi_{o}}\left|\sum_{x=a+1}^{a+B} \chi(x)\right|^{2 k} \ll_{\epsilon, k} \begin{cases}B^{k} p^{\epsilon}, & p^{1-\frac{1}{k}} \leq B<p, \\ p^{k-1+\epsilon}, & p^{\frac{5}{8}-\frac{1}{4 k}} \leq B \leq p^{1-\frac{1}{k}} \\ B^{k} p^{\frac{3}{8}(k-2)+\epsilon}, & p^{\frac{11}{24}} \leq B \leq p^{\frac{5}{8}-\frac{1}{4 k}} \quad(r=2), \\ B^{\frac{4}{3} k-\frac{2}{3}} p^{\frac{2}{9}(k-2)+\epsilon}, & p^{\frac{19}{48}} \leq B \leq p^{\frac{11}{24}} \quad(r=3), \\ \vdots & B \leq p^{1 / 4} .\end{cases}
$$

We have indicated (roughly speaking) the best upper bound available on each of the intervals in (17). 


\section{The Fundamental Identity And A Key Lemma}

View $\mathcal{B}$ and $V$ as subsets of $\mathbb{F}_{p}^{2 k}$, and let $\alpha$ denote the characteristic function of $\mathcal{B}$ with finite Fourier expansion

$$
\alpha(\mathbf{x})=\sum_{\mathbf{y}} a(\mathbf{y}) e_{p}(\mathbf{x} \cdot \mathbf{y}),
$$

where as usual $e_{p}(*)=e^{\frac{2 \pi i}{p} *}, \mathbf{x} \cdot \mathbf{y}=\sum_{i=1}^{2 k} x_{i} y_{i}, \sum_{\mathbf{y}}=\sum_{\mathbf{y} \in \mathbb{F}_{p}^{2 k}}$. The Fourier coefficients are given by

$$
a(\mathbf{y})=p^{-2 k} \prod_{i=1}^{2 k} e_{p}\left(-\left(a+\frac{1}{2}+\frac{B}{2}\right) y_{i}\right) \frac{\sin \left(\pi B y_{i} / p\right)}{\sin \left(\pi y_{i} / p\right)},
$$

where a term in the product is taken to be $B$ if $y_{i}=0$. We have

$$
\begin{aligned}
& \sum_{\chi \neq \chi_{o}}\left|\sum_{x=a+1}^{a+B} \chi(x)\right|^{2 k}=\sum_{\chi \neq \chi_{o}} \sum_{x_{1} \neq 0} \cdots \sum_{x_{2 k} \neq 0} \alpha(\mathbf{x}) \chi\left(x_{1} x_{2} \ldots x_{k} x_{k}^{-1} \ldots x_{2 k}^{-1}\right) \\
& =\sum_{\mathbf{y}} a(\mathbf{y}) \sum_{\chi \neq \chi_{o}} \sum_{x_{1} \neq 0} \cdots \sum_{x_{2 k} \neq 0} \chi\left(x_{1} x_{2} \ldots x_{k} x_{k+1}^{-1} \ldots x_{2 k}^{-1}\right) e_{p}(\mathbf{x} \cdot \mathbf{y}) \\
& =\sum_{\mathbf{y}} a(\mathbf{y}) \sum_{\chi \neq \chi_{o}} \prod_{i=1}^{k} \sum_{x_{i} \neq 0} \chi\left(x_{i}\right) e_{p}\left(x_{i} y_{i}\right) \prod_{i=k+1}^{2 k} \sum_{x_{i} \neq 0} \chi\left(x_{i}^{-1}\right) e_{p}\left(x_{i} y_{i}\right) .
\end{aligned}
$$

Now if $y_{i}=0$ for some $i$, then the sum over $x_{i}$ is zero, since $\chi$ is nonprincipal. If all of the $y_{i}$ are nonzero, then the sum over $\mathbf{x}$ is just

$$
\chi\left(\prod_{i=1}^{k} y_{i}^{-1} y_{i+k}\right) G(\chi)^{k} G\left(\chi^{-1}\right)^{k}=p^{k} \chi\left((-1)^{k} y_{1}^{-1} \ldots y_{k}^{-1} y_{k+1} \ldots y_{2 k}\right),
$$

where $G(\chi)$ denotes the Gaussian sum $G(\chi)=\sum_{x \neq 0} \chi(x) e_{p}(x)$. Here we have used the identities $G\left(\chi^{-1}\right)=\chi(-1) \overline{G(\chi)}$ and $|G(\chi)|^{2}=p$ for $\chi \neq \chi_{o}$. Summing over $\chi$ and using the identity,

$$
\sum_{\substack{y_{i} \neq 0 \\ \text { for all } i}} a(\mathbf{y})=p^{-2 k} \sum_{\mathbf{x} \in \mathcal{B}} \sum_{\substack{\mathbf{y} \\ y_{i} \neq 0}} e_{p}\left(x_{i} y_{i}\right)=\frac{B^{2 k}}{p^{2 k}}
$$

we obtain the

\section{Fundamental Identity.}

$$
\frac{1}{p-1} \sum_{\chi \neq \chi_{0}}\left|\sum_{x=a+1}^{a+B} \chi(x)\right|^{2 k}=p^{k} \sum_{\substack{y_{i} \neq 0 \\ y_{1} \cdots y_{k}=(-1)^{k} y_{k+1} \cdots y_{2 k}}} a(\mathbf{y})-\frac{B^{2 k}}{p^{k}(p-1)} .
$$

Lemma. Let $V^{ \pm} \subset \mathbb{Z}^{2 k}$ be the set of integer solutions of

$$
y_{1} \ldots y_{k} \equiv \pm y_{k+1} \ldots y_{2 k} \quad(\bmod p),
$$


and let $\mathcal{B}$ be the box of points $0<\left|y_{i}\right|<B_{i}, 1 \leq i \leq 2 k$, with the $B_{i}$ positive integers. Then

$$
\left|\mathcal{B} \cap V^{ \pm}\right| \ll_{\epsilon, k}\left(\frac{|\mathcal{B}|}{p}+\sqrt{|\mathcal{B}|}\right) p^{\epsilon}
$$

Proof. We may suppose without loss of generality that $\prod_{i=1}^{k} B_{i} \geq \prod_{i=k+1}^{2 k} B_{i}$ and that all of the $y_{i}$ are positive. Let $y_{k+1}, \ldots, y_{2 k}$ be any fixed values with $0<y_{i}<$ $B_{i}, k+1 \leq i \leq 2 k$, and put $c \equiv y_{k+1} \cdots y_{2 k}(\bmod p)$ with $0<c<p$. Then any integer solution $y_{1}, \ldots, y_{k}$ of $(24)$ with $0<y_{i}<B_{i}, 1 \leq i \leq k$, must satisfy

$$
y_{1} \cdots y_{k}=c+\ell p \quad \text { or } \quad y_{1} \cdots y_{k}=(p-c)+\ell p
$$

for some integer $\ell$ with $0 \leq \ell \leq \prod_{i=1}^{k} B_{i} / p$. For each such value $\ell$ the number of solutions of $(26)$ is $\leq(\tau(c+\ell p))^{k}+(\tau(p-c+\ell p))^{k} \ll_{\epsilon} p^{k^{2} \epsilon} \ll_{\epsilon, k} p^{\epsilon}$, where $\tau$ is the divisor function. Thus, the total number of solutions of (26) with $\ell$ in the specified range is

$$
\ll_{\epsilon, k}\left(\frac{\prod_{i=1}^{k} B_{i}}{p}+1\right) p^{\epsilon}
$$

We obtain the upper bound in (25) on multiplying by the number of choices for $y_{k+1}, \ldots, y_{2 k}$.

\section{Proof of the theOrem}

We start by noting that the Fourier coefficients (19) of the characteristic function $\alpha$ admit the upper bound

$$
|a(\mathbf{y})| \ll \prod_{i=1}^{2 k} \min \left(\frac{B}{p}, \frac{1}{\left|y_{i}\right|}\right) \quad\left(\left|y_{i}\right|<p / 2\right) .
$$

Letting the $y_{i}$ run through the intervals $0<\left|y_{i}\right| \leq p / B_{i}$ and $2^{r_{i}} p / B_{i}<\left|y_{i}\right| \leq$ $2^{r_{i}+1} p / B_{i}$ for $r_{i}=0,1,2, \ldots$, stopping when $2^{r_{i}}>B_{i} / 2$, we have

$$
\sum_{\substack{y_{i} \neq 0 \\ y_{1} \cdots y_{k}=(-1)^{k} y_{k+1} \cdots y_{2 k}}}|a(\mathbf{y})| \ll B^{2 k} p^{-2 k} \sum_{r_{1}=0} \cdots \sum_{r_{2 k}=0} \prod_{i=1}^{2 k} 2^{-r_{i}} \sum_{\substack{0<\left|y_{i}\right| \leq 2^{r_{i}} p / B \\ \mathbf{y} \in V^{ \pm}}} 1,
$$

where $V^{ \pm}$is as defined in the Lemma. Inserting the upper bound in (25), the above is

$$
\begin{gathered}
\ll_{\epsilon, k} B^{2 k} p^{-2 k} \sum_{r_{1}=0} \cdots \sum_{r_{2 k}=0} \prod_{i=1}^{2 k} 2^{-r_{i}}\left(\frac{p^{2 k-1}}{B^{2 k}} \prod_{i=1}^{2 k} 2^{r_{i}}+\frac{p^{k}}{B^{k}} \prod_{i=1}^{2 k} 2^{r_{i} / 2}\right) p^{\epsilon} \\
\ll_{\epsilon, k} p^{-1+\epsilon}+B^{k} p^{-k+\epsilon} .
\end{gathered}
$$

The theorem now follows immediately from the Fundamental Identity (23). 


\section{REFERENCES}

[1] A. Ayyad, T. Cochrane and Z. Zheng, The congruence $x_{1} x_{2} \equiv x_{3} x_{4}(\bmod p)$, the equation $x_{1} x_{2}=x_{3} x_{4}$ and mean values of character sums, J. Number Theory 59 (2) (1996), 398-413. MR 97i:11091

[2] D.A. Burgess, On character sums and L-series. II, Proc. London Math. Soc. (3) 13 (1963), 524-536. MR 26:6133

[3] H.L. Montgomery and R.C. Vaughan, Exponential sums with multiplicative coefficients, Inventiones Math. 43 (1977), 69-82. MR 56:15579

[4] H.L. Montgomery and R.C. Vaughan, Mean values of character sums, Canad. J. Math. 31 (3) (1979), 476-587. MR 81c:10043

Department of Mathematics, Kansas State University, Manhattan, Kansas 66506

E-mail address: cochrane@math.ksu.edu

Department of Mathematics, Zhongshan University, Guangzhou 510275, People’s RePUBLIC OF CHINA 